\title{
Response to Comment on "Pairing and Phase Separation in a Polarized Fermi Gas"
}

\author{
Guthrie B. Partridge, Wenhui Li, Ramsey I. Kamar, Yean-an Liao, and Randall G. Hulet \\ Sept 27, 2006

\begin{abstract}
Zwierlein and Ketterle rely on subjective arguments and fail to recognize important differences in physical parameters between our experiment and
\end{abstract} \\ theirs. We stand by the conclusions of our original report.
}

Having failed to establish that trap anharmonicities or other objective mechanisms affect the conclusions of our study [1], Zwierlein and Ketterle [2] now make the more subjective assertion that our claims are not supported by the data presented. We stand by the statements and claims made in [1]. Furthermore, in emphasizing discrepancies between our results and theirs, Zwierlein and Ketterle ignore differences in physical parameters of the two experiments. Differences in aspect ratio [3] or temperature [4,5], for example, can have profound effects on the results.

1. G. B. Partridge, Wenhui Li, R. I. Kamar, Y. A. Liao, and R. G. Hulet, Science 311, 503 (2006). Published online 21 December 2005 (10.1126/science.1122876).

2. M. Zwierlein and W. Ketterle, preceding Comment.

3. T. N. De Silva and E. J. Mueller, Phys. Rev. Lett. 97, 070402 (2006).

4. K. B. Gubbels, M. W. J. Romans, and H. T. C. Stoof, cond-mat/0606330 (2006).

5. M. M. Parish, F. M. Marchetti, A. Lamacraft, and B. D. Simons, cond-mat/0605744 (2006). 